\title{
Calibration of accelerometers by shock excitation and laser interferometry ${ }^{1}$
}

\author{
Alfred Link and Hans-Jürgen von Martens \\ Physikalisch-Technische Bundesanstalt (PTB), \\ Abbestrasse 2-12, 10587 Berlin, Germany \\ Received 25 May 1999 \\ Revised 22 July 1999
}

A signal processing method for determining the dynamic behavior of accelerometers by shock excitation and laser interferometry is presented. The method allows the shock sensitivity and the magnitude and phase lag of the complex sensitivity of accelerometers to be accurately measured. After digitizing the phase-modulated signals at the output of a homodyne or heterodyne interferometer, the time-dependent displacement is reconstructed on the basis of the principle of coherent demodulation. Data processing developed for determining peak value and spectrum of the acceleration efficiently suppresses disturbing quantities. Computer simulations and experiments proved that the shock parameters can be measured with expanded uncertainties of less than $0.2 \%$. Based on the investigation reported, the method has been adopted by the ISO/TC 108/SC 3 as a primary shock calibration method to be specified in a new international standard ISO 16063-13 "Primary shock calibration using laser interferometry".

Keywords: Quadrature signal, digital signal processing, shock, accelerometer, interferometer, calibration

\section{Introduction}

Measurements of shocks and other transient accelerations are carried out on a large scale in various fields of sensor applications (e.g. airbag accelerometer, motor knock sensors, measurement techniques in impact testing of road vehicles, instrumentation for environmental

\footnotetext{
${ }^{1}$ An earlier form of this paper was presented at the 1st International Conference on Vibration Measurements by Laser Techniques, Ancona, Italy, October 1994. The conference was organized by the Italian Association of Laser Velocimetry. The articles were selected, edited and reviewed by a committee chaired by: Professor Enrico Primo Tomasini, Department of Mechanics, Faculty of Engineering, University of Ancon, Italy.
}

testing). The calibration of shock and vibration measuring instruments consists in determining the relationship of the output to the input, i.e., determining its sensitivity. According to the relevant international standard ISO 16063-1:1998 (revised and renumbered), the frequency response of accelerometers is defined as complex sensitivity $S_{a}=\left(\left|U_{A C}\right| /|A|\right) \exp j\left(\varphi_{A}-\varphi_{A C}\right)$, and the output-to-input ratio of peak values as shock sensitivity $S_{s h}=u_{A C, m} / a_{m} \cdot A(j \omega)$ and $a_{m}$ denote spectrum and peak value of the input acceleration, whereas $U_{A C}(j \omega)$ and $u_{A C, m}$ denote spectrum and peak value of the accelerometer output. To measure the input acceleration with high accuracy, laser interferometry (homodyne and heterodyne) can be used. Laser interferometers provide output signals whose phase signal $\varphi(t)$ is proportional to the displacement $s(t)$ to be measured, i.e. $\varphi(t)=4 \pi \lambda^{-1} s(t)$, where $\lambda$ is the wavelength of the laser light.

With the development of special shock exciters for generating rectilinear shock motion, and of different interferometric shock measurement methods, the Physikalisch-Technische Bundesanstalt (PTB) has responded to the requirement that traceability be established for the measurements of shocks. The shock calibration facilities of the PTB allow highly accurate absolute calibration of accelerometers which are then used for the calibration of shock measurement instrumentation by the comparison method.

To generate shock acceleration in a peak value range from 100 to $5000 \mathrm{~m} / \mathrm{s}^{2}$, a special shock exciter has been developed. It is fitted with an airborne hammer and an airborne anvil to which the accelerometer to be calibrated is attached [1,2]. A modified Michelson interferometer is used for measuring the displacement of an excited shock motion. It provides two output signals phase-shifted by $90^{\circ}$ (quadrature signals). Since the shape of the generated acceleration is nearly half a sine squared wave, the model introduced in this contribution is called sine squared impulse.

To generate shock acceleration at higher peak values of up to $10^{5} \mathrm{~m} / \mathrm{s}^{2}$, wave propagation in a long thin bar is used. A heterodyne interferometer specially modified 
for this purpose is part of this measuring system. It provides an output signal whose spectrum is located in the high-frequency range around the heterodyne carrier frequency of about $40 \mathrm{MHz}$. The acceleration shape of the generated shock can be approximately described by the derivative of a Gaussian function. For simplification, the introduced impulse model is called Gaussian impulse. The shock exciter developed allows to generate acceleration impulses of short duration, which are used to perform measurements of the frequency response of accelerometers in a frequency range from $1 \mathrm{kHz}$ to $30 \mathrm{kHz}$.

This contribution describes signal processing procedures for reconstructing the phase signal and computing peak value and spectrum of shock acceleration. The proposed signal processing method is based on quadrature signals generated by either optical means or digital signal processing. Computer simulations and experimental investigations have shown that peak value and spectral components can be measured with expanded uncertainties of less than $0.2 \%$.

\section{Processing scheme}

Figure 1 shows the signal processing scheme for computing the shock sensitivity $S_{s h}$ and the complex sensitivity $S_{a}$ of accelerometers calibrated by shock acceleration. The displacement of an acceleration impulse excited by a shock machine is measured by an interferometer. The interferometer converts the continuous-time displacement $s(t)$ into the phase signal $\varphi(t)=4 \pi \lambda^{-1} s(t)$. The interferometer output signal is a phase-modulated electrical signal furnished by light detectors. Homodyne interferometers are equipped with two light detectors, which provide two output signals phase-shifted by $90^{\circ}$. Heterodyne interferometers provide a phase-modulated output signal with the heterodyne carrier frequency $\omega_{h}$. After analog-to-digital (A/D) conversion of the interferometer output signal, a coherent demodulation procedure is applied to obtain the phase signal $\varphi(t)$ at instants of time $t_{n}, n=1,2, \ldots$. Acceleration characteristics of interest are the peak value and the spectrum of the second derivative $d^{2} \varphi(t) / d t^{2}$. They are obtained using signal processing procedures as described below.

The signal processing of the accelerometer output signal involves only two stages: A/D conversion and computation of peak value and spectrum. For example, the peak value can be obtained by applying an algorithm comparing successive signal samples to detect the maximum value of a sequence. The acceleration spectrum is defined as discrete-time Fourier transform (DFT), which can be computed by applying fast Fourier transform (FFT) algorithms. Both, the algorithm for detecting a peak value and the FFT algorithm are standard signal processing procedures which will not be explained in the following.

We have to note that the output signal of an accelerometer is understood here as the output signal of a connected amplifier. In measuring practice, the amplifier is utilized for transforming the high output impedance of the accelerometer into the low impedance of connected measuring systems. It is clear that the frequency response of the accelerometer is influenced by the amplifier frequency response. In general, the influence of the upper cut-off frequency of the amplifier does not lead to difficulties in applying signal processing procedures for the computation of peak value and spectrum. In contrast to this, the lower cut-off frequency causes a slowly varying time signal, which corresponds to an exponential decay $\exp (t / \tau)$, where $\tau$ is the time constant of this process. Defining the lower cut-off frequency as $f_{L}=1 /(2 \pi \tau)$ and presupposing a measuring time $T_{M} \ll \tau$, we find that $f_{L}<0.00016 / T_{M}$ provides a relative signal decay smaller than $10^{-3}$ at the end of the measuring interval. In most cases, errors caused by such a slowly varying decay can be neglected when peak value and spectrum are computed. In the following, we presuppose that the lower cut-off frequency of the amplifier has been chosen to meet this requirement.

\section{Test signals}

Sine squared impulse and Gaussian impulse are two test signals frequently used to determine the input/output relation of an accelerometer. The acceleration of a sine squared impulse is described by

$$
\begin{aligned}
& a_{\sin }(t)=a_{\sin , m} \sin ^{2}\left(\pi \frac{t}{T_{\sin }}\right), \\
& 0 \leqslant t \leqslant T_{\sin } .
\end{aligned}
$$

Solving the integral of Eq. (1) we obtain the velocity function

$$
\nu_{\sin }(t)=a_{\sin , m}\left[\frac{t}{2}-\frac{T_{\sin }}{4 \pi} \sin \left(2 \pi \frac{t}{T_{\sin }}\right)\right] .
$$

For modeling a Gaussian impulse, we use the wellknown Gaussian function

$$
\nu_{G}(t)=\nu_{G, m} e^{-\frac{1}{2}\left(\frac{t-t_{0}}{T_{G}}\right)^{2}}, 0 \leqslant t \leqslant T_{d}
$$




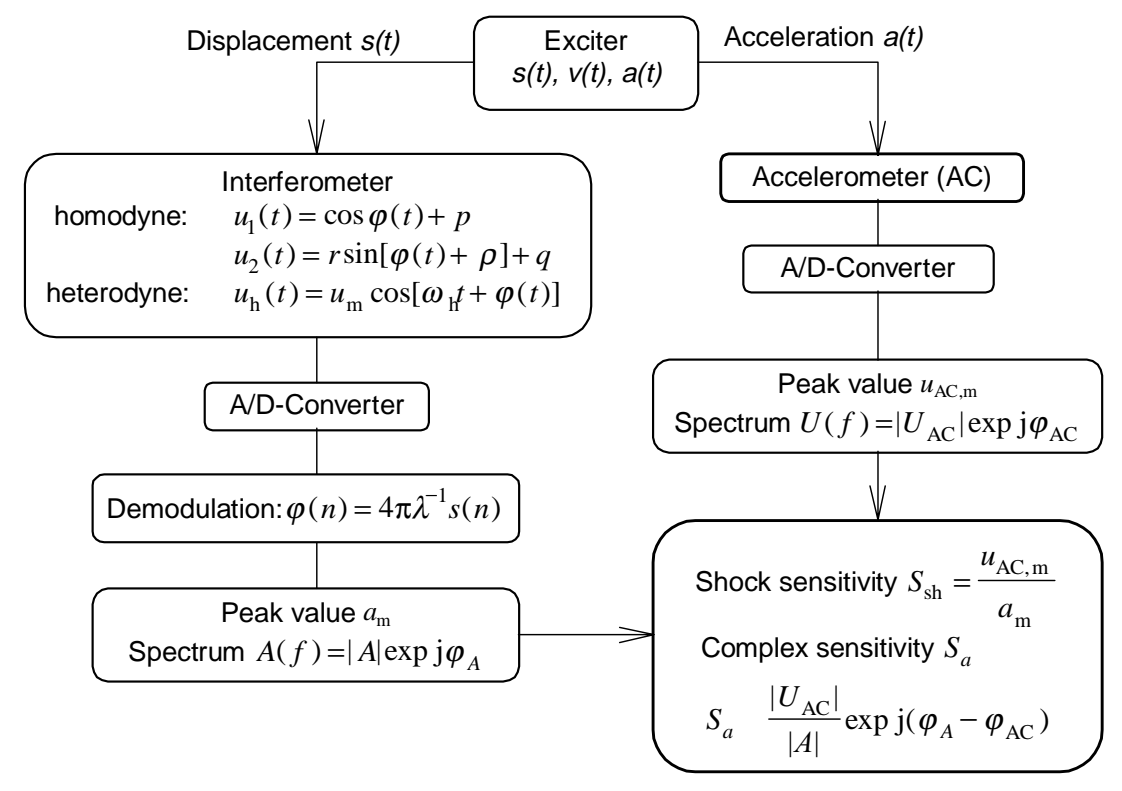

Fig. 1. Signal processing scheme for determining shock sensitivity and complex sensitivity.

which describes the velocity curve of the Gaussian impulse. Taking the derivative of Eq. (3) we obtain the acceleration as

$$
a_{G}(t)=-\frac{\nu_{G, m}}{T_{G}^{2}}\left(t-t_{0}\right) e^{-\frac{1}{2}\left(\frac{t-t_{0}}{T_{G}}\right)^{2}} .
$$

The acceleration curve is characterized by two extreme values

$$
a_{G, m}=\frac{\nu_{G, m}}{\sqrt{e} \cdot T_{G}}
$$

located on the time axis at $t=t_{0} \pm T_{G}$. Figure 2 illustrates acceleration, velocity and displacement of the models introduced. In practice, a sine squared impulse is typically realized with a duration $T_{\sin }>$ $1 \mathrm{~ms}$. The Gaussian impulse is characterized by a parameter ratio $T_{G} / T_{d}$ in the range from 0.05 to 0.2 . The maximum velocity value of the Gaussian impulse is located on the time axis at $t_{0}$. In relation to the impulse duration $T_{d}$, we observe a ratio $t_{0} / T_{d}$ usually ranging from 0.2 to 0.3 . The impulse duration of generated Gaussian impulses is about $1 \mathrm{~ms}$.

In contrast to generated sine squared impulses, the energy of a Gaussian impulse is approximately concentrated in a small time interval $2 T_{G}$. This interval can be understood as impulse width of the Gaussian impulse. Since the relationship between the interferometer phase signal and the corresponding displacement is given by $\varphi(t)=4 \phi \lambda^{-1} s(t)$, we obtain velocity or acceleration curves of a measured phase signal by multiplying the instantaneous frequency $d \varphi / d t$ or the second deriva- tive of the phase $d^{2} \varphi(t) / d t^{2}$ by the factor $(4 \pi / \lambda)^{-1}$. To obtain estimates of the bandwidth (given in radian frequency) of the modeled acceleration impulses, we use

$$
B_{\mathrm{sin}}^{(L F)}=\frac{2 \pi}{T_{\mathrm{sin}}}
$$

for a sine squared impulse and

$$
B_{G}^{(L F)}=\frac{\pi}{T_{G}}
$$

for a Gaussian impulse. In contrast to these estimates, the bandwidth of the corresponding modulated interferometer signals is substantially larger. As a good approximation we can use the expressions

$$
\left(\frac{d \varphi}{d t}\right)_{\max }=B_{\sin }^{(H F)}=\frac{2 \pi}{\lambda} a_{\sin , m} T_{\sin }
$$

for a sine squared impulse and

$$
\left(\frac{d \varphi}{d t}\right)_{\max }=B_{G}^{(H F)}=\frac{4 \pi}{\lambda} \sqrt{e} a_{G, m} T_{G}
$$

for a Gaussian impulse. The $B^{(H F)} / B^{(L F)}$ ratio of the frequency bandwidths is a measure of the possible bandwidth reduction when demodulating the interferometer signals. Using Eqs. (6)-(9) we obtain

$$
\frac{B_{\sin }^{(H F)}}{B_{\sin }^{(L F)}}=\lambda^{-1} a_{\sin , m} T_{\sin }^{2}
$$

and 

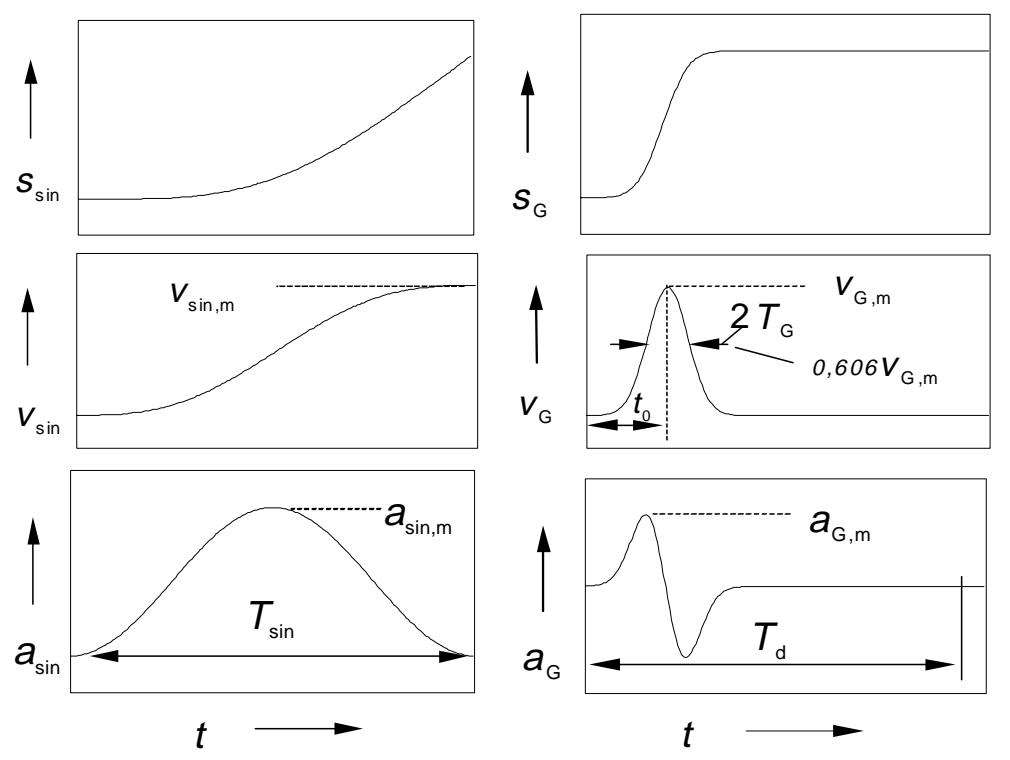

Fig. 2. Displacement $s(t)$, velocity $v(t)$ and acceleration $a(t)$ curves. (Left) Sine squared impulse and (right) Gaussian impulse.

$$
\frac{B_{G}^{(H F)}}{B_{G}^{(L F)}}=2 \sqrt{e} \lambda^{-1} a_{G, m} T_{G}^{2} .
$$

Equations (10) and (11) show the dependence of the possible bandwidth reduction on peak value and impulse width.

According to the Nyquist sampling theorem, the sampling frequency of the interferometer signals has to be chosen to fulfill the condition

$$
2 \pi f_{s}>2 B^{(H F)},
$$

whereas, in the case of heterodyne interferometer signals, we must ensure that $2 \pi f_{s}>4 \omega_{h}$ is fulfilled $\left(\omega_{h}>B^{(H F)}\right.$ is presupposed).

\section{Coherent demodulation}

Homodyne interferometer signals can be described [3] as

$$
u_{1}(t)=\cos \varphi(t)+p
$$

and

$$
u_{2}(t)=r \sin [\varphi(t)+\rho]+q .
$$

The quadrature signals $u_{1}(t)$ and $u_{2}(t)$ contain the phase signal $\varphi(t)$ which is given by $\varphi(t)=4 \pi \lambda^{-1} s(t)$. Mostly, when measuring the displacement of an acceleration impulse, we can neglect disturbing motions. Therefore, in the following, such disturbing motions will not be explicitly introduced into Eqs. (13) and (14).
On the contrary, signal degradation caused by non-ideal generation of a quadrature signal by means of optical and analog electrical devices has to be taken into account. This signal degradation is described by the parameters $r, p, q$ and $\rho$, where $r$ stands for different amplifier gains, $p$ and $q$ are offset errors and $\rho$ is the phase shift error between the quadrature signals. We assume that these parameters can be considered as constanttime values for the measuring time $T_{M}$. Generally, the values of these disturbing parameters do not exceed the limits

$$
\begin{aligned}
& 0.85<r<1.15,|p, q|<0.1 \text { and } \\
& |\rho|<0.2 \mathrm{rad} .
\end{aligned}
$$

Heterodyne interferometers provide a phase-modulated signal in the form

$$
u_{h}(t)=u_{m} \cos \left[\omega_{h} t+\varphi(t)\right]
$$

When multiplying $u_{h}(t)$ by quadrature carrier signals $\sin \omega_{h} t$ and $\cos \omega_{h} t$ and performing low-pass filtering $\left(\mathrm{LP}_{h}\right)$ with a cut-off frequency $\omega_{c}^{(H F)} \approx B^{(H F)}$, we obtain base-band quadrature signals. In contrast to quadrature signals generated by optical means, heterodyne quadrature signals are affected only by negligibly small disturbing signals because of the high accuracy achieved by digital signal processing.

Figure 3 shows the coherent demodulation scheme. Since we can assume that the phase-modulated interferometer signals can be considered complicated signals, the input signals of the coherent demodula- 


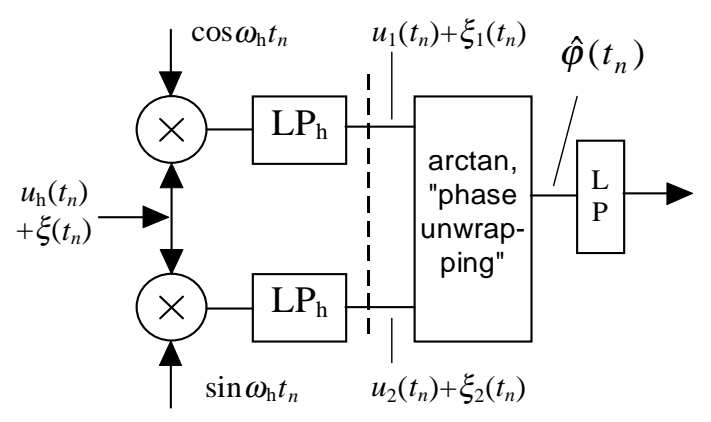

Fig. 3. Coherent demodulation scheme. The left side applies only to heterodyne interferometry.

tion scheme are represented as discrete-time signal at $t_{n}, n=0,1,2, \ldots$, superposed by an additive noise process $\xi\left(t_{n}\right)$, i.e. the quantization noise samples of the preceding A/D conversion are thought of as an additive noise signal. The left side of the demodulation scheme is related to the generation of quadrature signals by digital signal processing. For quadrature signals generated by optical means, only the right side of the scheme is valid. Applying an arctangent subroutine to the base-band quadrature signals and using a phase unwrapping procedure, we obtain an estimate of the phase sequence

$$
\begin{aligned}
& \hat{\varphi}\left(t_{n}\right)=\arctan \frac{u_{2}\left(t_{n}\right)}{u_{1}\left(t_{n}\right)}+\chi \pi, \\
& \chi=0,1,2, \ldots
\end{aligned}
$$

The succeeding low-pass filter is used to attenuate disturbing signals situated outside the frequency range from 0 to $B^{(L F)}$.

It is known [4] that for complicated signals the quantization error $\xi\left(t_{n}\right)$ can be considered as an uncorrelated noise process. Moreover, the error sequence will not be correlated with the sampled interferometer sequences $u_{1}(t), u_{2}(t)$, or $u_{h}(t)$, if heterodyne interferometry is made use of. In particular, the assumptions of this statistical model appear to be valid if the heterodyne signal $u_{h}(t)$ is asynchronously sampled, i.e. $f_{s} \neq(l / g) f_{h}$ and $l, g$ are integers. If the peak signal amplitude of interferometer signals is matched to the full-scale range of a $(b+1)$-bit quantizer, an estimate of the noise variance of the low-pass filter phase output signal will be [5]

$$
\sigma_{\varphi}^{2}=\frac{2^{-2 b} \omega_{c}}{12 \pi f_{s}},
$$

where $\omega_{c}$ is the radian cut-off frequency of the lowpass filter. For example, if the sampling frequency is $f_{s}=100 \mathrm{MHz}$, the bandwidth of the output signal is reduced to $f_{c}=100 \mathrm{kHz}$, and if $b=5$ is realized, we obtain $\sigma_{\varphi}<0.001 \mathrm{rad}$.

Deterministic phase errors are caused by non-ideal quadrature signals, i.e. quadrature signals affected by $r, p, q$ and $\rho$ parameters $(r \neq 1)$. If $z_{0}(t)=$ $\cos \varphi(t)+j \sin \varphi(t)$ describes the vector of an ideal quadrature signal and the vector $z(t)=\cos \varphi(t)+$ $p+j[r \sin (\varphi(t)+\rho)+q]$ represents a really generated quadrature signal, an estimate of the deterministic phase error is given by [6]

$$
\begin{aligned}
& \Delta \varphi(t)=q \cos \varphi(t)-p \sin \varphi(t)+r \sin \rho \\
& \cos ^{2} \varphi(t)+(r \cos \rho-1) \sin \varphi(t) \cos \varphi(t) .
\end{aligned}
$$

Analysis of Eq. (19) reveals that width and amplitude of the phase error spectrum depend on instantaneous frequency $d \varphi / d t$ and $r, p, q$ and $\rho$ values. Relative high-frequency spectral components can be attenuated by the low-pass filter arranged behind. Thus, we can expect that a suitable choice of the cut-off frequency of the low-pass filter will allow sufficient performance (accuracy) to be achieved for the computation of the peak value or the spectrum of the acceleration. If quadrature signals generated by optical means are applied, according to the limits specified by (15), the degradation of the phase sequence is dominated by deterministic phase errors.

\section{Peak value determination}

The design of a signal processing procedure for determining the peak value involves two basic steps: the realization of the second derivative of the phase (or displacement) sequence, and the estimation of the peak value of the acceleration sequence derived. The signal processing procedure realizing an input/output relation of a second derivative is designed as a cascade structure consisting of two differentiators and low-pass filters. Figure 4 shows a block diagram of basic operations for computing the peak value. The phase sequence $\varphi(n)$ is the input. The first low-pass filter is that of the coherent demodulation scheme. The output of the first differentiator (D) provides samples of the instantaneous frequency (or velocity), and the output samples of the second differentiator are samples of the acceleration signal. To ensure an optimal choice of the signal processing bandwidth, each differentiator cascade is preceded by a low-pass filter.

Given the acceleration sequence, a computer subroutine $(\mathrm{PV})$ is used, which provides the maximum value 


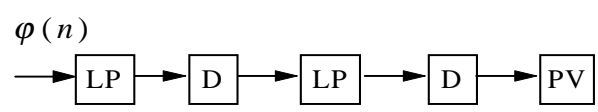

Fig. 4. Block scheme representation of peak value determination. LP - low-pass filter, D - differentiator, PV - peak value detection.

of a data sequence. The smoothness of the acceleration sequence allows to apply a simple algorithm comparing successive samples without any approximation procedure. In the following analysis, we will discuss only the first basic step, i.e. the design of a low-pass filter and a differentiator to obtain samples of the derivative of a band-limited input signal.

If the derivative of a data sequence is defined as the slope of a quadratic curve, fitting three data points $n-1, n$ and $n+1$, the slope will be obtained by the signal processing algorithm

$$
y(n)=0.5[x(n+1)+x(n-1)],
$$

where $x(n)$ is the input and $y(n)$ the output. This signal processing procedure is characterized by the frequency response

$$
H(j \Omega)=j \sin \Omega,
$$

where $\Omega$ denotes the normalized radian frequency. From Eq. (21) it can be seen that this is a linear phase differentiator which amplifies high-frequency signal components up to and beyond $\Omega=0.5 \pi$. To prevent high-frequency disturbing signal components from becoming stronger in the differentiator output spectrum, it is necessary to introduce attenuation at frequencies above critical frequency. This can be accomplished by low-pass filtering of the input signal. The choice of the cut-off frequency of a low-pass filter means to determine such a critical frequency. As peak value determination is mostly applied to a sine squared impulse, this acceleration impulse may serve as an example to demonstrate the method for the low-pass filter design. Using a linear approximation of the velocity curve within the time region of the peak value of the corresponding acceleration signal, we can write

$$
\frac{d \varphi}{d t} \cong \frac{4 \pi}{\lambda} a_{\sin , m} t=\gamma t .
$$

Applying a low-pass filtering procedure of the first order with a continuous-time system function [7]

$$
G(s)=\frac{\omega_{c}}{s+\omega_{c}},
$$

we obtain the filter output signal

$$
y(t)=\omega_{c}^{-1} \gamma\left(e^{-\omega_{c} t}-1\right)+\gamma t,
$$

where $\omega_{c}$ denotes the cut-off filter frequency. From Eq. (24) it can be seen that the behavior of the output signal is linear, when $t>\omega_{c}$. Suppose that we wish to achieve this filter behavior for $t>T_{\sin } / K$ with $K>1$. On the other hand, we wish to design a low-pass filter realizing a specified attenuation around $t=0.5 T_{\text {sin }}$. For example, the filter has to attenuate deterministic disturbing signals (see Eq. 8) at frequencies

$$
\frac{d \varphi}{d t}\left(t=0.5 T_{\sin }\right)=\frac{2 \pi}{\lambda} a_{\sin , m} T_{\sin }=\omega_{\Delta \varphi}
$$

Suppose that the low-pass filters are designed to meet this specification by $M=\omega_{\Delta \varphi} / \omega_{c}$ with $M>1$. Combining the two filter specifications needed, we obtain the relationship

$$
k / T_{\sin }<\omega_{c}<\omega_{\Delta \varphi} / M,
$$

which furnishes the lower and upper limits of the cut-off frequency,

$$
\omega_{c 1}=K / T_{\sin } \text { and } \omega_{c 2}=\frac{2 \pi}{M \lambda} a_{\sin , m} T_{\sin }
$$

Figure 5 illustrates the relationship (26) for $M=10$ and $K=100$, where $a_{\sin , m}$ was chosen as a parameter. It can be seen that, if the impulse duration $T_{\sin }$ is reduced, the choice of possible $\omega_{c}$ values will be confined to a smaller interval, because the difference between the upper and lower limits of the cut-off frequency decreases. Using a low-pass filter of higher order we can reduce the $M$ value. However, in this case, we have to take into account that high-order filters are characterized by a longer filter response. Computer simulation and experimental results have shown that a Butterworth filter of fourth order can in most cases meet the filter specifications, realizing a tradeoff between an attenuation of disturbing signals and a small distortion of the acceleration signal.

Figure 6 illustrates simulation results obtained for a sine squared impulse with a duration $T_{\sin }=5 \mathrm{~ms}$ and peak values from $a_{\sin , m}=100$ to $1000 \mathrm{~m} / \mathrm{s}^{2}$. Simulated quadrature signals were quantized to 6 bit $(b=5)$ and generated as non-ideal quadrature signals characterized by errors, which complied with the tolerances specified above (15). For deriving the acceleration signal from the input phase samples we have used differentiators as explained above in connection with fourth order Butterworth low-pass filters specified by a cutoff frequency $f_{c}=10 / T_{\sin }=2 \mathrm{kHz}$. In Fig. 6, the simulation results show that peak values were obtained with relative errors $\Delta a_{\sin , m} / a_{\sin , m}$ smaller than $10^{-3}$. On the basis of these results, the method proposed for determining the peak value can be regarded as robust as regards disturbing signals caused by optical generation of quadrature signals. 


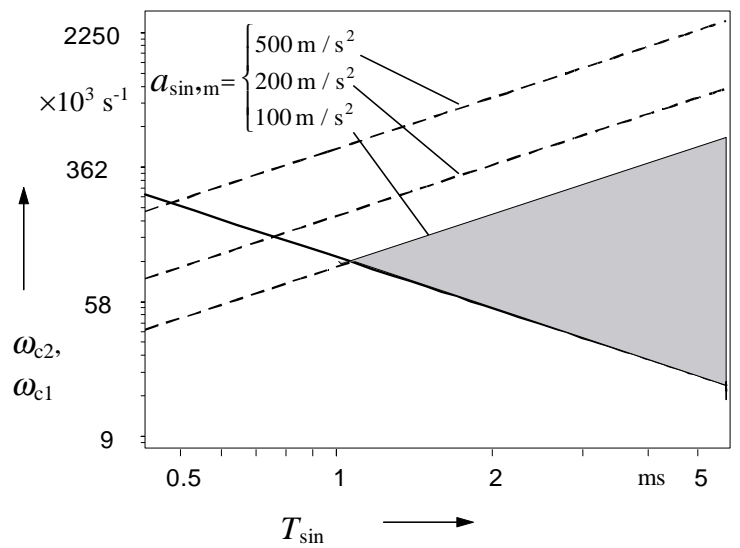

Fig. 5. Lower (solid) and upper (dashed) limits of the low-pass filter cut-off frequency (Eq. 27) as a function of the impulse duration $T_{\sin }$.

\section{Computation of the acceleration spectrum}

To obtain the frequency response of an accelerometer in a broad frequency range, we should use an exciting acceleration impulse whose signal energy is concentrated on an interval which is small compared with the impulse duration. When a Gaussian impulse is used a small $T_{G} / T_{d}$ ratio should be realized. The following analysis presupposes that the measuring time $T_{M}$ is equal to the impulse duration $T_{d}$.

The acceleration spectrum of a displacement or phase impulse $x(t)$ can be obtained by calculating the Fourier transform $F\left\{x^{\prime \prime}(t)\right\}$ of the second derivative of the displacement signal $x^{\prime \prime}(t)$. Using the known relationship

$$
\begin{aligned}
& F\left\{x^{\prime}(t)\right\}=\int_{0}^{T_{M}} x^{\prime}(t) \exp (-j \omega) d t=x(t) \\
& \left.\exp (-j \omega t)\right|_{0} ^{T_{M}}+j \omega \int_{0}^{T_{M}} x(t) \exp (-j \omega t) d t
\end{aligned}
$$

we can express the Fourier transform of the derivative as

$$
F\left\{x^{\prime}(t)\right\}=j \omega F\{x(t)\},
$$

where the condition

$$
x(0)=x\left(T_{M}\right)=0
$$

must be fulfilled. From Fig. 2 we see that the condition of Eq. (30) holds only for the velocity curve of the Gaussian impulse model. However, to satisfy the condition of Eq. (30), we can apply the Fourier transform to the sheared signal

$$
x_{\text {she }}(t)=x(t)+g(t)
$$

where $g(t)$ is a linear shearing function determined by $x_{\text {she }}(0)=x_{\text {she }}\left(T_{M}\right)=0$. Clearly, the contribu- tion of $g(t)$ to the spectrum $F\left\{x_{\text {she }}^{\prime \prime}(t)\right\}$ obtained after twice applying Eq. (29) vanishes and $F\left\{x_{\text {she }}^{\prime \prime}(t)\right\}=$ $F\left\{x^{\prime \prime}(t)\right\}$ holds. Equally, the first derivative of the displacement signal can be calculated in the time domain. The resulting velocity curve satisfying the condition of Eq. (30), can be transformed into the frequency domain. Here, we will analyze the later way. Figure 7 shows the corresponding signal processing scheme. After lowpass filtering (LP) and differentiating (D) the phase sequence $\varphi(n)$, we obtain samples of the instantaneous frequency or velocity. As Fig. 2 illustrates, the velocity curve starts and ends at a level of nearly zero. Computing the discrete Fourier transform (DFT) of the velocity signal we can expect to obtain spectral components with a negligibly small error due to a leakage effect of the Fourier transform. Therefore, performing the second differentiating operation by multiplying the velocity spectral components by $j \omega$, we obtain the acceleration spectrum.

The input low-pass filter (LP) in Fig. 7 is that of the coherent demodulation scheme. Choosing a value of the filter cut-off frequency which corresponds to the bandwidth of the acceleration signal, we can achieve a minimal noise bandwidth for the first differentiation operation in the time domain. Since the bandwidth of the Gaussian impulse is inversely proportional to parameter $T_{G}$, we define the filter cut-off frequency as

$$
f_{c} \leqslant \frac{5}{T_{G}} \quad \text { or normalized as } \quad f_{c}^{*}=\frac{f_{c}}{f_{s}},
$$

where $f_{s}$ is the sampling frequency given by Eq. (12).

In the following analysis, we denote the $k$-th spectral component of the acceleration spectrum by $A(k)=$ $|A(k)| \exp (j \varphi(k))$, and define a range of DFT values by $k=1, \ldots, k_{E}$, which is characterized by amplitude values $|A(k)|>0.1|A|_{\max }$, where $|A|_{\max }$ is the maximum amplitude value of the acceleration spectrum.

To prove relation (32), computer simulations were carried out. We observed that the relative error $|\triangle A(k)| /|A(k)|$ is smaller than $10^{-4}$ within the range from 1 to $k_{E}$, if relation (32) is fulfilled.

The influence of quantization noise on the determination of the acceleration spectrum can be estimated when, for example, a uniformly distributed white noise signal is added to the phase sequence $\varphi(n)$. A common measure of the degradation of a signal by additive noise is the signal-to-noise ratio (SNR) defined as the ratio of signal variance (power) to noise (variance) [4]. To quantify the SNR we have calculated the power of the harmonic signal content of the phase or displacement signal of the Gaussian impulse. Figure 8(a) and 


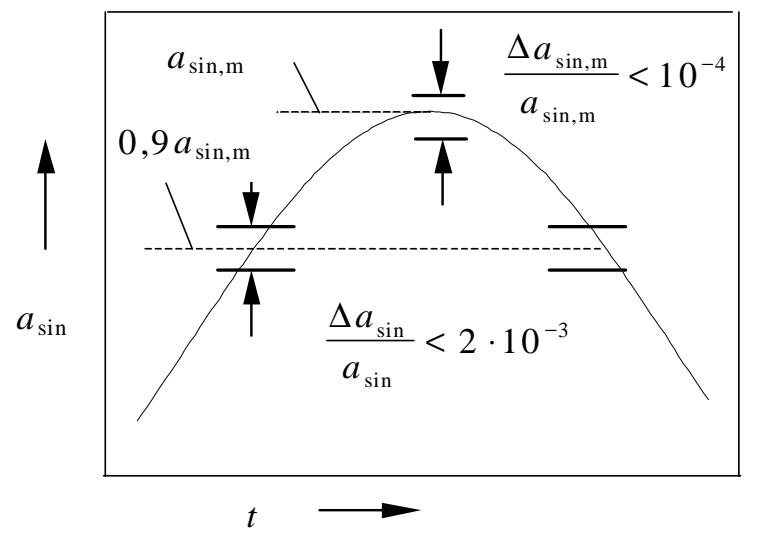

Fig. 6. Simulation results obtained for a sine squared impulse with a duration $T_{\sin }=5 \mathrm{~ms}$ and peak values in the range from $a_{\sin }=100$ to $1000 \mathrm{~m} / \mathrm{s}^{2}$. Quadrature signals are quantized to $b=5$ and disturbed by $0.85<r<1.15 ;|p, q|<0.1 ;|\rho|<0.2 \mathrm{rad}$.

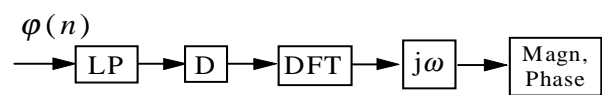

Fig. 7. Block scheme of signal processing for computing the acceleration spectrum. LP - low-pass filter, D - differentiator, DFT - discrete-time Fourier transform, Mag, Phase - calculation of magnitude and phase.

(b) show simulation results of the calculated acceleration spectrum when the phase sequence is contaminated by noise. These results were obtained for a Gaussian impulse characterized by a ratio $t_{0} / T_{d}=0.25$. As normalized measuring time, a ratio $0.5 T_{d} / T_{G}$ is used. For Gaussian impulses normalized in this way, relevant spectral components were found at $k=1$ up to $k_{E}=8$. A cut-off frequency of $f_{c}^{*}=0.1$ of the low-pass filter was chosen. Amplitude errors $\Delta A(k)$ and phase errors $\Delta \varphi_{A}(k)$ were calculated in relation to spectral components obtained for noise-free phase samples. Figure 8(a) shows the standard deviation of the relative amplitude values, $s(A)=\sqrt{\left.<(|\Delta A| /|A|)^{2}\right\rangle}$, and Figure $8(\mathrm{~b})$ shows the standard deviation of the phase values, $s\left(\varphi_{A}\right)=\sqrt{\left\langle\left(\Delta \varphi_{A}\right)^{2}\right\rangle}$, for SNRs 63, 51 and $43 \mathrm{~dB}$ (traces 1, 2 and 3). If quadrature signals are quantized to $b>4$, we can expect an SNR of the phase sequence above $40 \mathrm{~dB}$ [5]. When the simulation results given in Figs. (8) are taken into account we can expect that even the influence of a small quantizing accuracy will cause errors in the order of only $10^{-3}$ in magnitude measurements.

Deterministic disturbing signals described by Eq. (19) provide a broadband disturbing spectrum, as it is known for phase-modulated signals with a large modulation
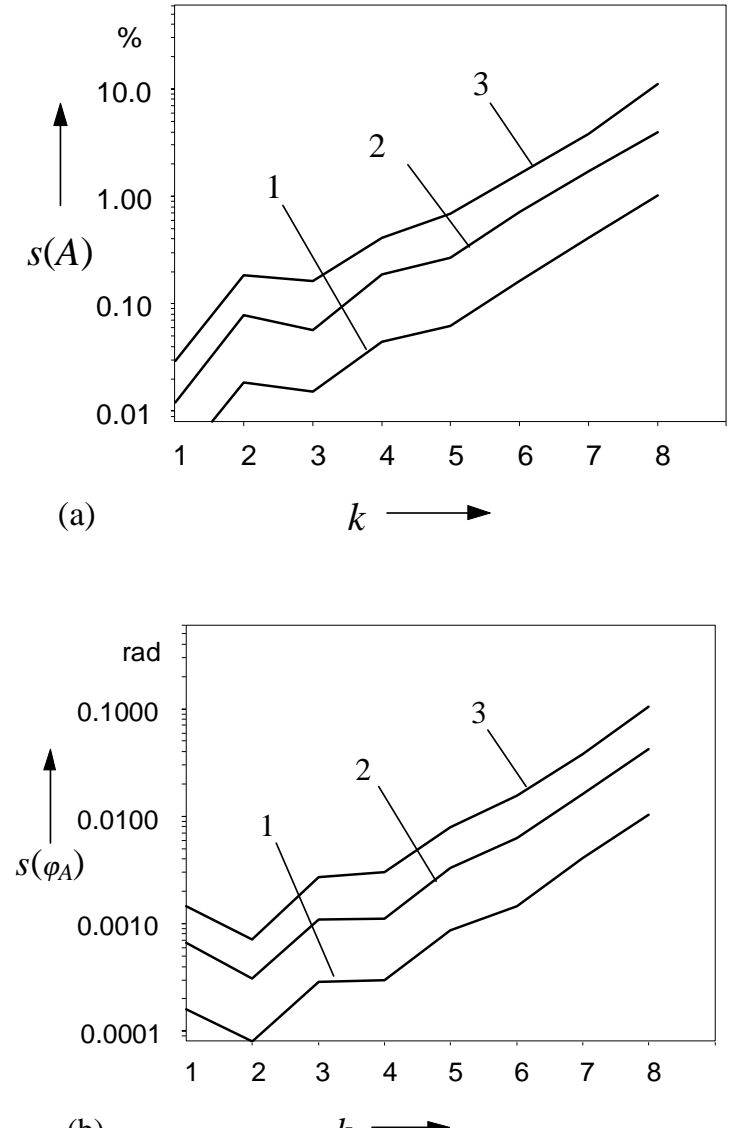

(b)

Fig. 8. (a) Relative amplitude standard deviation, $s(\mathrm{~A})$, and (b) standard deviation of phase values, $s(\varphi \mathrm{A})$, of the acceleration spectrum obtained for contamination of the phase sequence $\varphi(n)$ by noise. Traces 1,2 and 3 correspond to $\mathrm{SNR}=63,51$ and $43 \mathrm{~dB} . k-\mathrm{DFT}$ frequency sample number.

index. Low-frequency components of this spectrum mainly cause errors when the acceleration spectrum is computed. Calculating the integral of Eq. (3) we see that phase samples are proportional to both the maximum value of the acceleration impulse and the impulse width. We therefore can expect that the influence of disturbing signals is inversely proportional to these impulse parameters. For the normalized Gaussian impulse chosen above with $t_{0} / T_{d}=0.25$ and $k_{E}=8$, this influence could be demonstrated for maximum values $a_{G, m}=10^{4} \mathrm{~m} / \mathrm{s}^{2}$ and $10^{3} \mathrm{~m} / \mathrm{s}^{2}$. Figure 9 (a) and (b) show simulation results for $a_{G, m}=10^{4} \mathrm{~m} / \mathrm{s}^{2}$. Phase shift errors were simulated with $|\rho|=0.2,0.1$, and $0.01 \mathrm{rad}$ (traces 1, 2 and 3, respectively). The results were calculated in relation to phase samples without any phase shift error. It can be seen that even for $|\rho|=0.2 \mathrm{rad}$ the relative amplitude error $|\Delta A| /|A|$ is 

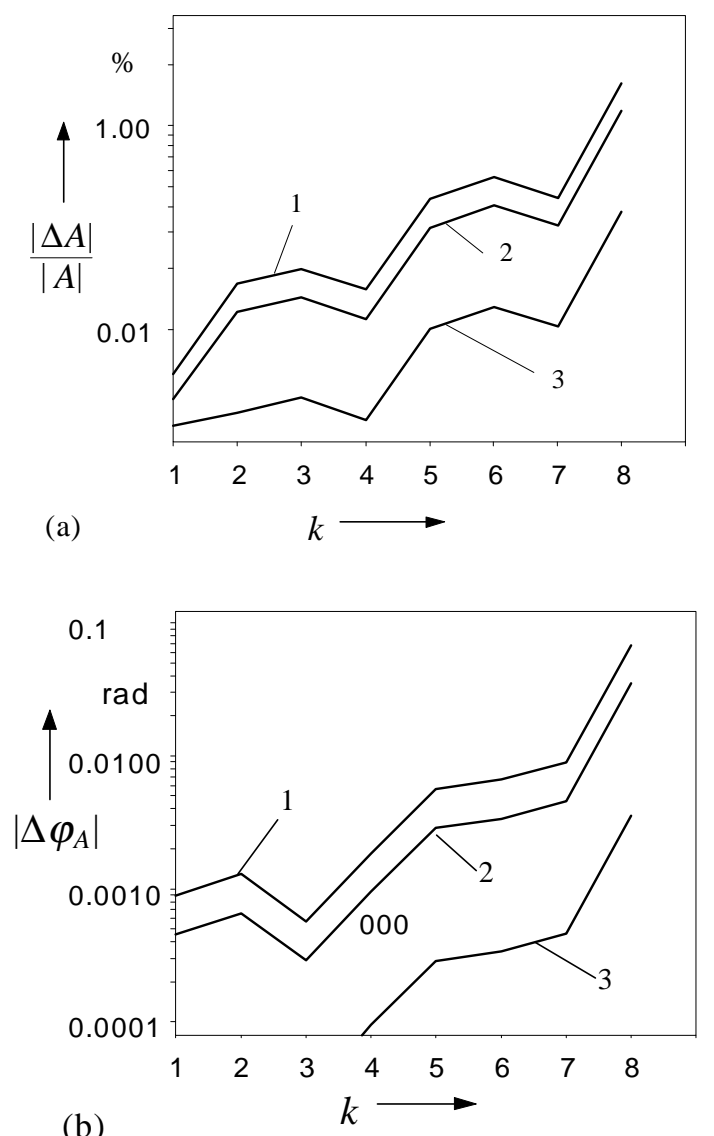

Fig. 9. (a) Relative amplitude error $|\Delta A| /|A|$ and (b) phase error $\left|\Delta \varphi_{A}\right|$ of the acceleration spectrum obtained for non-ideally generated quadrature signalswith $|\rho|=0.2,0.1$ and $0.01 \mathrm{rad}$ (trace 1,2 and 3). Impulse parameters: $a_{G, m}=10^{4} \mathrm{~m} / \mathrm{s}^{2}, T_{G}=50 \mu \mathrm{s}$.

smaller than $0.2 \%$ and the phase error $\left|\Delta \varphi_{A}\right|$ smaller than $0.01 \mathrm{rad}$ within the range $k=1, \ldots, 5$. If disturbing signals are caused by offset errors which lie within the tolerance range (15), the relative amplitude errors are below $0.2 \%$ and phase errors below $0.015 \mathrm{rad}$ $(k=1, \ldots, 5)$. In the case of $a_{G, m}=10^{3} \mathrm{~m} / \mathrm{s}^{2}$, the relative influence of disturbing signals on the acceleration spectrum is however greater. For $|\rho|=0.2,0.1$, and $0.01 \mathrm{rad}$ compared with $a_{G, m}=10^{4} \mathrm{~m} / \mathrm{s}^{2}$, the relative amplitude errors are doubled and phase errors are ten times as high. When quadrature signals affected by offset and gain errors were simulated, we found that their influence is substantially greater than that of a phase shift error on the calculation of the acceleration spectrum. For example (see Fig. 10), when $|q|=0.1$ was chosen, we observed a relative amplitude error in the order of some percent, whereas the phase errors were smaller than 0.004 rad within the range of $k=1, \ldots, 6$.
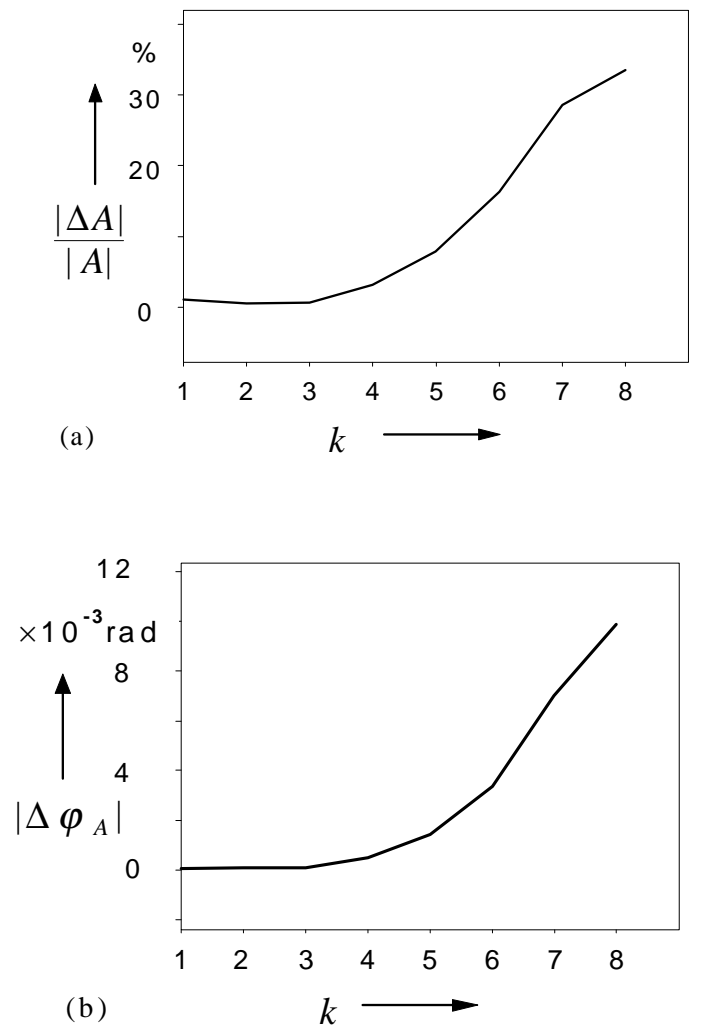

Fig. 10. (a) Relative amplitude error $|\Delta A| /|A|$ and (b) phase error $\left|\Delta \varphi_{A}\right|$ of the acceleration spectrum obtained for non-ideally generated quadrature signals with $|q|=0.1$. Impulse parameters: $a_{G, m}=10^{3} \mathrm{~m} / \mathrm{s}^{2}, T_{G}=50 \mu \mathrm{s}$.

By the simulation of disturbing signals originating from quantizing and from the non-ideal generation of quadrature signals, we have shown their characteristic influence on the computation of the acceleration spectrum. Relatively great errors occur when the phase sequence $\varphi(n)$ is contaminated by deterministic disturbing signals caused by $r, \rho, p$ and $q$ errors. Such errors typically occur when quadrature signals are generated by optical means. We therefore conclude that measurements using quadrature signals generated by optical means are basically less accurate than those using quadrature signals obtained by signal processing. Generally, when quadrature signals generated by digital signal processing are applied, disturbing signals are widely suppressed. We therefore should prefer measurements using the generation of quadrature signals by digital signal processing if the exciting Gaussian impulse is characterized by a maximum value $a_{G, m}$ below $10^{4} \mathrm{~m} / \mathrm{s}^{2}$ and an impulse width $T_{G}$ smaller than $50 \mu \mathrm{s}$. 


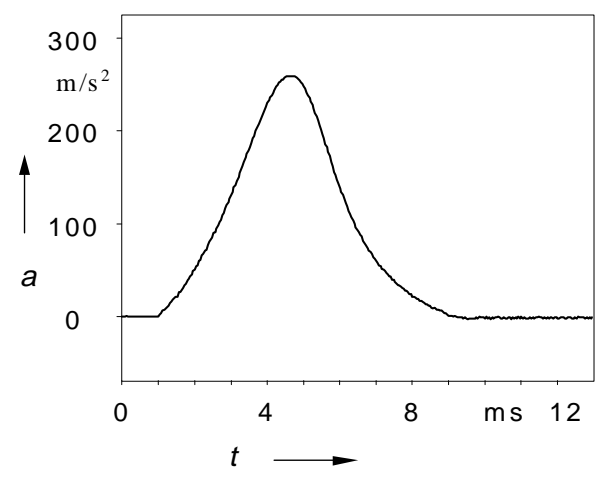

Fig. 11. Acceleration curve of a measured sine squared impulse.

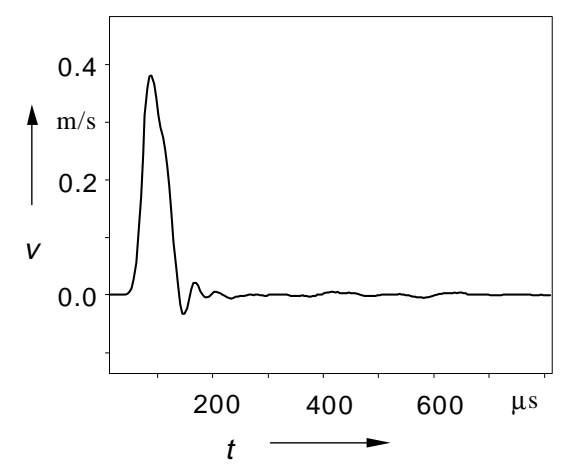

Fig. 12. Velocity curve of a measured high-level shock (Gaussian impulse).

\section{Experimental results}

The proposed signal processing method was experimentally investigated and verified in measurements of the shock sensitivity $S_{s h}$ and the complex sensitivity $S_{a}$ of commercial accelerometers. Both, the interferometer output signal and the accelerometer output signal were digitized by means of an 8-bit A/D converter.

Sine squared impulse accelerations were provided by a special shock exciter as described above. The modified Michelson interferometer connected provides quadrature output signals. Figure 11 shows an acceleration impulse generated by the shock exciter.

It can be seen that a half-sine squared function can be used to model this impulse form. In order to evaluate the method for computing the impulse peak value, a commercial accelerometer was tested in a test series consisting of six shock impulses with $a_{\sin , m}$ of about $300 \mathrm{~m} / \mathrm{s}^{2}$. The impulse duration was about $7 \mathrm{~ms}$. This means that the impulse spectrum is located below $500 \mathrm{~Hz}$. Since the resonant frequency of the accelerometer tested was higher than $30 \mathrm{kHz}$, the shock sensitivity $S_{s h}$ corresponds to the complex sensitivity of the accelerometer at low frequencies. When computing the peak values of the accelerometer input and output signals, we obtained a mean value of the shock sensitivity for this test series which was in good agreement with the complex sensitivity measured by a sinusoidal calibration method at $80 \mathrm{~Hz}$. The relative difference of mean values between $S_{s h}$ and $S_{a}(f=80 \mathrm{~Hz})$ was smaller than $0.13 \%$. The relative standard deviation computed for the six single test shocks was $0.15 \%$. A variety of measurements including this example has confirmed that the signal processing method proposed for determining the peak value of sine squared acceleration impulses can be used for computing the shock sensitivity of accelerometers when quadrature signals are generated by optical means.

For the generation of shock accelerations at highlevel peak values of up to $10^{5} \mathrm{~m} / \mathrm{s}^{2}$, wave propagation in a long thin bar (Hopkinson bar [8]) is applied. Using the shock exciter developed at the PTB, we have calibrated a commercial accelerometer by acceleration shocks with peak values of about $2.5 \cdot 10^{4} \mathrm{~m} / \mathrm{s}^{2}$. The displacement of the shock impulses generated was measured by a specifically modified commercial heterodyne interferometer. Interferometer and accelerometer output signals were synchronously sampled with a sampling frequency $f_{s}=100 \mathrm{MHz}$.

Figure 12 shows a velocity curve of a generated shock. This example demonstrates that the Gaussian impulse model introduced can be used as an approximation to these high-level shocks. The resonant frequency of the mounted accelerometer was greater than $40 \mathrm{kHz}$. The relevant spectral content of the exciting acceleration impulse ranged from below $2 \mathrm{kHz}$ to $10 \mathrm{kHz}$. Spectral components at higher frequencies exhibited amplitude values smaller than 0.1 of the maximum value located at about $4 \mathrm{kHz}$. When setting a measuring time $T_{M}=0.5 \mathrm{~ms}$ we obtain equidistantly spaced spectral components at $2,4, \ldots, 10 \mathrm{kHz}$ etc.

Using the signal processing method developed above for the reconstruction of the phase (displacement) sequence and the computation of the acceleration spectrum, and applying the discrete Fourier transform to the output signal of the accelerometer, we obtain the input and output spectra of the accelerometer for determining its complex sensitivity. To calculate the complex sensitivity we have made use of the cross-spectrum method according to Bendat and Piersol [9]. Using the terminology given in [9] we define auto-spectra and crossspectra by $G_{x x}, G_{y y}, G_{x y}$ and $G_{y x}$, where indices $x$ stand for the input acceleration impulse and $y$ for the accelerometer response data. Presupposing indepen- 

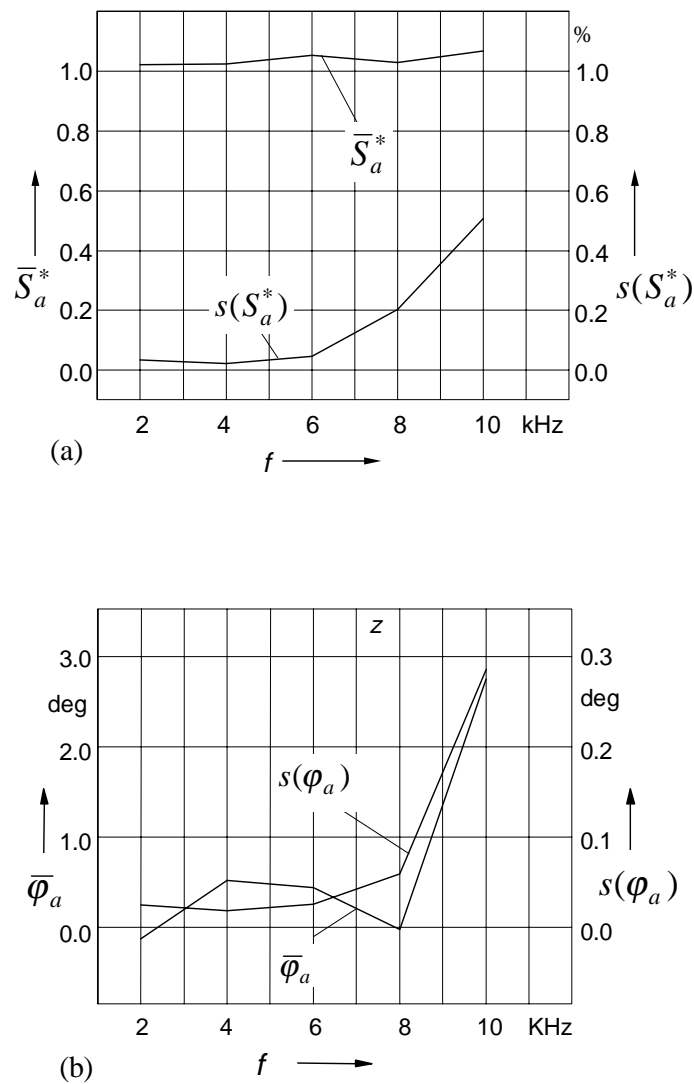

Fig. 13. Mean and standard deviation of the complex sensitivity of a commercial accelerometer (obtained from seven test shocks with $a_{G, m} \sim 2.5 \cdot 10^{4} \mathrm{~m} / \mathrm{s}^{2}$ ). (a) mean $\overline{S_{a}^{*}}$ and standard deviation $s\left(S_{a}^{*}\right)$ of the magnitude and (b) mean $\overline{\varphi_{a}}$ and standard deviation $s\left(\varphi_{a}\right)$ of the phase lag.

dent output noise, we have calculated the complex sensitivity using the equation

$$
S_{a}(f)=\frac{\sum G_{x y}(f)}{\sum G_{x x}(f)},
$$

where the summations are performed for the ensemble of seven shock pulses entered into the accelerometer at a particular shock level and pulse duration. The coherence, $\gamma_{x y}^{2}(j \omega)$, can be expressed as

$$
\gamma_{x y}^{2}(f)=\frac{\left|\sum G_{x y}(f)\right|^{2}}{\sum G_{x x}(f) \sum G_{y y}(f)} .
$$

It was also calculated for the ensemble of data sets. For the ensemble of seven shock pulses, we found that the coherence was greater than 0.99 up to frequencies below $10 \mathrm{kHz}$. This value shows that only low-level disturbing signals were encountered in the measurements.

To characterize the distribution of the complex sensitivities obtained for single shocks, the mean and stan- dard deviations of the complex sensitivities of single input shocks were also calculated.

Figure 13(a) shows the mean $\bar{S}_{a}^{*}$ and the standard deviation $s\left(S_{a}^{*}\right)$ of the magnitude of the complex sensitivity, and Fig. 13(b) shows the mean $\bar{\varphi}_{a}$ and the standard deviation $s\left(\varphi_{a}\right)$ of the phase lag of the complex sensitivity. Here, to simplify the notation, we used normalized magnitude values denoted by *. As can be seen in Fig. 13(a), in the frequency range from 2 to $8 \mathrm{kHz}$, for example, we obtained relative standard deviation values smaller than $0.2 \%$. The standard deviation increases towards higher frequencies, as can be expected from the small spectral amplitudes of the exciting impulses in this range.

\section{Conclusions}

The results of the theoretical and experimental investigations show that the signal processing method allows high accuracy (low measurement uncertainty) to be achieved as required for the shock calibration of precision-grade accelerometers in the frequency range from $2 \mathrm{kHz}$ to $20 \mathrm{kHz}$. Since acceleration peak values can be generated and interferometrically measured up to at least $10^{5} \mathrm{~m} / \mathrm{s}^{2}$ with an expanded uncertainty (coverage factor of 2 ) of $0.2 \%$, the sensitivity of accelerometers over a broad peak value range of interest can be determined (with an expanded uncertainty: $0.5 \%$ to $1 \%$ ). In conjunction with high-intensity acceleration excitation, the signal processing method is therefore used at the PTB for analyzing the effect of the nonlinearity of accelerometers and impact measuring instrumentation [10].

The state of the art achieved by the method is demonstrated in [11] for a calibration of an accelerometer using sinusoidal vibration with an amplitude of $100 \mathrm{~m} / \mathrm{s}^{2}$ and shock excitation in the peak value range from $1000 \mathrm{~m} / \mathrm{s}^{2}$ to $65000 \mathrm{~m} / \mathrm{s}^{2}$. No significant difference occurred between sinusoidal vibration calibration and low-level shock calibration (agreement within 0.1\%), and the good consistency between the shock calibration results at different levels confirm the specified expanded uncertainty of $0.5 \%$ to $1 \%$ (level-dependent) of the absolute shock calibrations. ISO TC 108/SC 3 "Use and calibration of vibration and shock measuring instruments" has recognized the suitability of the method as a primary shock calibration method, having adopted it as standard method to be specified in the new international standard ISO 16063-13 "Primary shock calibration using laser interferometry" (current stage: Committee Draft). 


\section{References}

[1] B. Glöckner, H.-J. v. Martens, P. Rogazewski, H.-J. Schlaak, A. Täubner and W. Wabinski, Neue Verfahren zur Kalibrierung von Stoßmeßmitteln, Metrologische Abhandlungen 8(3) (1988), 245-258.

[2] H.-J. v. Martens, H.-J. Schlaak and A. Täubner, Interferometrische Kalibrierung von Translations- und Rotationsaufnehmern mit Sinus- und Stoßanregung, Fortschritte der Akustik, DAGA 94, part B, Bad Honnef: DPG GmbH, 1994, pp. 681-684.

[3] P.L.M. Heydemann, Determination and correction of quadrature fringe measurement errors in interferometers, Applied Optics 20(19) (1981), 3382-3384.

[4] V. Oppenheim and R.W. Schafer, Discrete-time signal processing, Prentice Hall, Englewood Cliffs, NJ, 1989.

[5] A. Link, Zum Signal-Rausch-Verhältnis digitalisierter Interferometer-Meßsignale bei kohärenter Demodulation
(Technisches Messen, under prep.).

[6] A. Link and H.-J. v. Martens, Amplitude and phase measurement of sinusoidal vibration in the nanometer range using laser interferometry, Measurement 24 (1998), 55-67.

[7] H. Marko, Systemtheorie, Springer Verlag, 1995.

[8] H. Kolsky, Stress waves in solids, Dover Publication, New York, 1963.

[9] J.S. Bendat and A.G. Piersol, Engineering Applications of correlation and spectral analysis, John Wiley \& Sons, 1980.

[10] H.-J.v. Martens, G. Lerche, A. Link, A. Täubner and W. Wabinski, Kalibrierung von Beschleunigungsaufnehmern durch Stoßwellen-Anregung und Laser-Interferometrie, Fortschritte der Akustik, DAGA 97, Bad Honnef: DPG GmbH, 1997.

[11] H.-J.v. Martens, Current state and trends of ensuring traceability for vibration and shock measurements, Metrologia 38 (1999), (to be printed). 

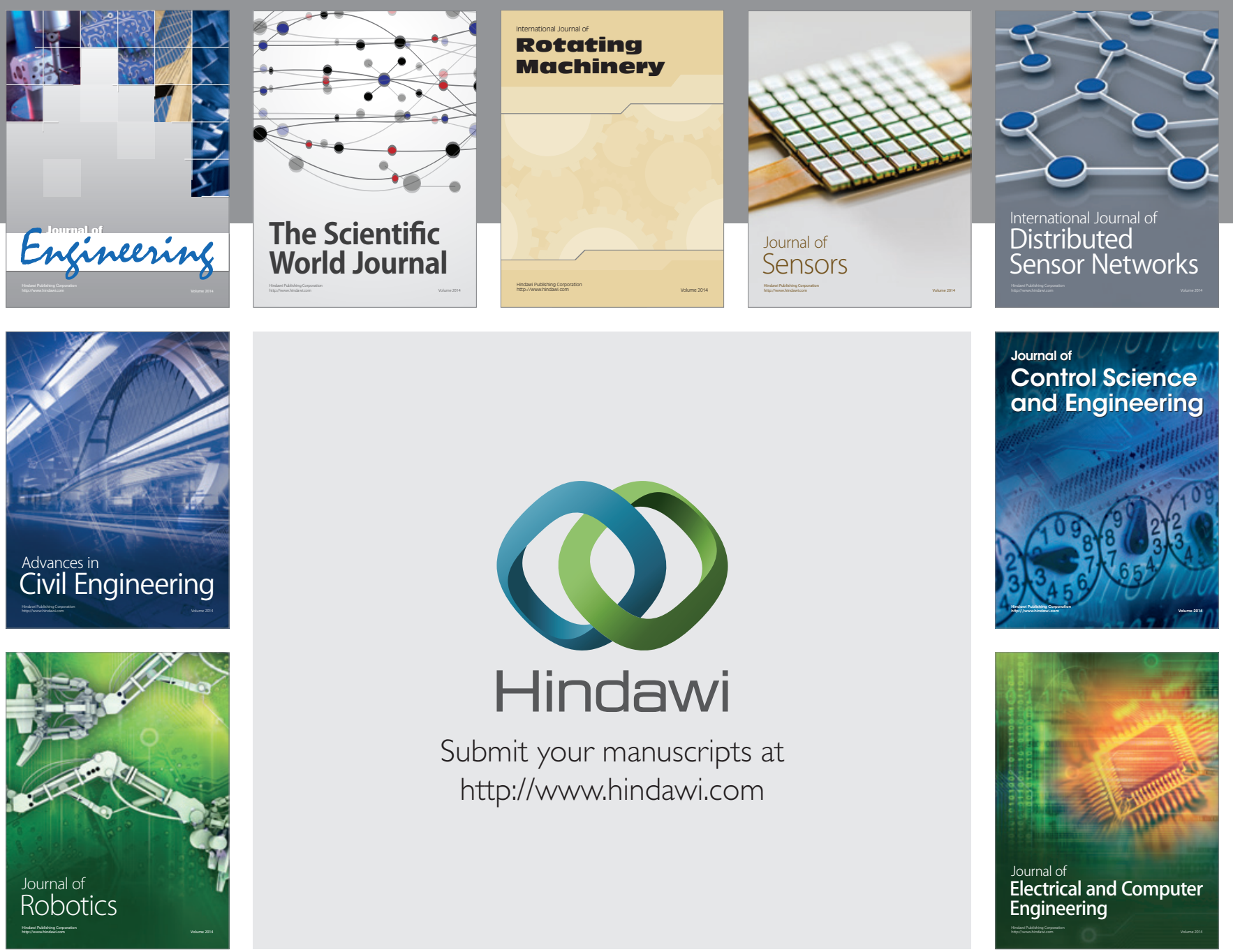

Submit your manuscripts at

http://www.hindawi.com
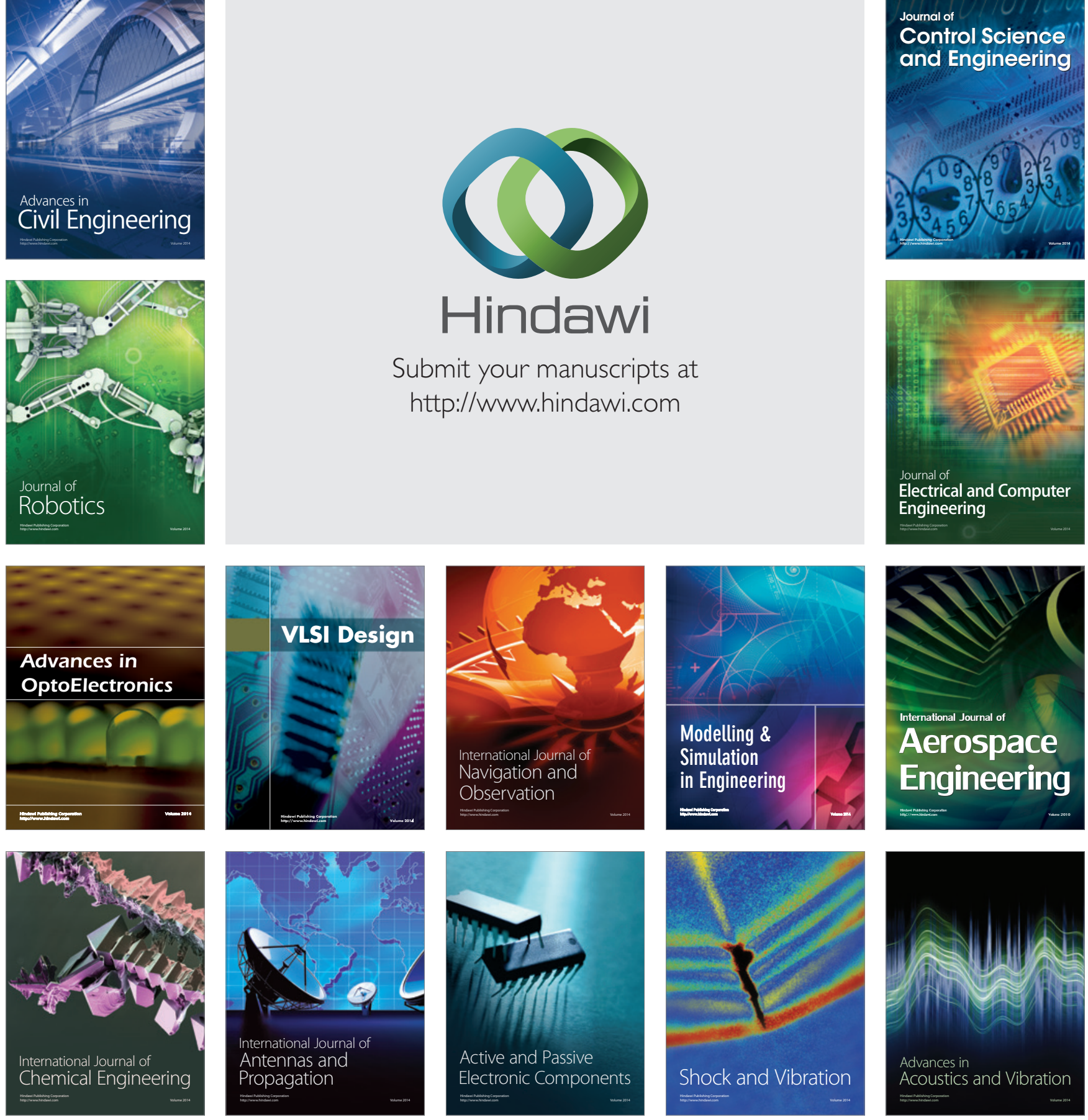